Whitworth Digital Commons

Whitworth University

Library Faculty Scholarship

Library

7-7-2015

\title{
User Preferences and Library Space at Whitworth University Library
}

Paul Ojennus

Whitworth University, pojennus@whitworth.edu

Kathy A. Watts

Whitworth University, kwatts@whitworth.edu

Follow this and additional works at: http:// digitalcommons.whitworth.edu/libraryfaculty

Part of the Library and Information Science Commons

\section{Recommended Citation}

Ojennus, P., \& Watts, K.A. (2015). User preferences and library space at Whitworth University Library. Journal of Librarianship and Information Science, published online before print July 7, 2015. doi: 10.1177/0961000615592947

This Article is brought to you for free and open access by the Library at Whitworth University. It has been accepted for inclusion in Library Faculty Scholarship by an authorized administrator of Whitworth University. 
User preferences and library space at Whitworth University Library

\section{Introduction}

Changes in the ways patrons use libraries over the last twenty years have affected library design requirements, and at the same time user-groups have become increasingly diverse, with the result that design principles tend to be formulated at a highly abstract level and individual solutions need to be formulated on a case-by-case basis. Harriet Cheney Cowles Memorial Library at Whitworth University was designed and built in 1992 with a vision that was still largely oriented toward print collections. Given the changes in resource format and access since 1992, we knew our building's design was no longer the best fit for the needs of current students and faculty. Recent trends in academic libraries to introduce coffee shops or large common study areas, to reduce print resources in favor of electronic, or to move print resources off-site present intriguing ideas to meet current user needs. Before embarking on any changes, however, we needed to understand our user group better, specifically ways in which our user group is similar to national trends and where it differs. We decided, therefore, to survey our user-group to quantify how they used our current facilities, whether they identified themselves as likely to benefit from the suggested renovations, and to explore whether there were other needs that we had not yet identified.

After reviewing the literature, we saw that while other libraries had conducted similar surveys on the subject of renovations, no survey had yet addressed a user-group closely comparable to ours. The literature review established many of the core areas that patrons were interested in having improved, such as technology, hours/access, study areas, aesthetics, and amenities, and also showed that user needs could vary widely depending on the context of the library. Therefore, our primary goals in the survey were to establish that there was a real need for improving our facilities and to prioritize any such improvements according to user needs. Since our student body had a history of high response rates to online questionnaires, we decided to conduct the survey in that way, using the Qualtrics survey software already set up by our Office of Institutional Research.

\section{Literature review}

A number of trends in Library and Information Science research helped establish the topics that were likely to be of interest to our user-group; building on these, we could use our survey to identify priorities particular to our patrons from among those trends. First, the review confirmed that the focus of library space has changed from collections to access and learning, but that this continues to be a matter of degree, so that physical collections continue to have importance, depending on the user-group. Second, the literature repeatedly pointed out that there are few universal rules for library facilities, but that the context, mission, and user needs 
should determine individual solutions. Third, surveys identified core areas of patron interest, such as technology, hours/access, study areas, aesthetics, and amenities. Finally, the literature review suggested that the idea of the library as space could be used to build or reinforce a certain culture among the patrons, but that this, again, needed to be consistent with the mission of the library and user needs.

\section{From collections to access and productivity}

Particularly because of the advent of electronic formats for many traditional library resources over the last 20 years, the focus of the library has changed from housing and preserving resources to providing access and spaces for learning, while facilities planning decisions must align themselves with these orientations (Stewart, 2010). Latimer (2010) identifies the factors driving this change in orientation as the increasing availability of electronic resources, the shift in balance between print and electronic resources, technological advancements such as self-issue, social networking, and increasing competition from other information providers such as Google. Latimer (2011) reviews the history of library design and how individual building projects in academic libraries have responded to these changes in orientation. She highlights a number of trends centering on the issue of access: accommodating IT demands, shelving solutions (compact shelving, off-site storage) for less-used print collections, sustainability, aesthetics, and development of social spaces.

While the focus of libraries is shifting away from print collections, providing access to these materials continues to be an important task; the approach a library takes to providing access to print collections should be tailored to the needs of its user-group. For example, Burton and Kattau (2013) describe Macquarie University Library's decision to adopt an automated storage and retrieval system in preference to other shelving solutions such as mass weeding, off-site storage, or compact shelving. The decision to split the print collections between open shelving for high-use items and automated storage and retrieval for low-use and legacy items was based on the needs of the library's users: selections for the open shelves largely corresponded to the teaching and learning needs of the undergraduate population, while the legacy collections were retained because of their usefulness for faculty research. Academic departments were consulted throughout the process to keep the location determinations aligned to the needs of the patrons. At the other end of the spectrum, Vaska, Chan, and Powelson (2009) found their health science students relied primarily on electronic resources and journals, and so they opted for rigorous weeding of low-circulation monographs and removing older journals to off-site storage. May and Swabey (2015) found that half of the students they surveyed across five academic libraries reported searching for information by browsing the shelves and concluded that the use of print collections varied significantly among the libraries they examined, depending on several variables, such as program type (bachelor's 
degree vs. technical and vocational programs), size of collections, and library design. Overall, we can identify the main trends in the shift from collections to access as reducing open shelving by reducing collections or various technological solutions, and providing technology to facilitate access to electronic collections. The degree of this shift, however, varies by the context of individual libraries.

\section{Diversity of user-groups}

At the same time, academic libraries have become much more attuned to the variations in their individual contexts and how they can respond to the changing needs of their patrons. The second key theme we identified in the review of the literature was the diversity among user-groups, and how their individual characteristics shaped how they used library spaces. The literature on this theme is still developing, with some scholars primarily noting the need for such attention, and others addressing specific contexts, such as the kind of institution, academic pursuits of students, the role of commuter students, and gender- and ethnicityspecific library behavior. Nitecki (2011: 54) identifies as an important area for further research, "How will libraries create and improve learning spaces to address the specific needs of local constituents without falling into the trap of simply emulating what others have done, thus missing an opportunity for the library to engage the larger learning and research agendas of its institution," and Stewart (2010: 11) similarly admonishes, "User groups differ in their expectations of the library as a communal space." Again, Black and Roberts (2006) warn against stereotyping students as uniformly "net generation", and demonstrate the need to accommodate variation within user-groups. May and Swabey (2015) identified the type of institution academic libraries served (community college, undergraduate university, or technical institute) as an important influence on how users employed library spaces. Latimer (2010) identifies characteristics of library design that are pertinent to art and design students, such as

group spaces for cross-disciplinary work, appropriate shelving, and good quality design. Burton and Kattau (2013) identify current teaching and research focuses of the university and publishing trends (e.g. that ancient history continues to favor print format) as determinants of how to divide library space between open shelves and automated retrieval collections. BrownSica (2012) points to demographic factors such as urban context, ethnic and international diversity, and residential/commuter-student mix as influencing the use of the library, for example, the preponderance of commuter-students at Auraria Library correlated to heavy demands for study spaces and an eating area. Similarly, Applegate (2009) correlates an overwhelming off-campus population to the decision to turn over library space to computer workstations at Indiana University Purdue University Indianapolis Library. Applegate (2009), Given and Leckie (2003), Mozingo (1989), and DeClecq and Cranz (2014) identify differences in uses of library space along gender lines. Yoo-Lee, Lee, and Velez (2013) observe correlation between ethnic background and uses of library spaces. 
Recognizing that ideas for updates or improvements to the library could come from observing successful projects targeted at other user-groups, and that the literature substantiated that different user-groups had different needs in terms of library space, we saw that it was necessary to prioritize our improvements by the needs of our patrons, as Lippincott (2010) recommends. In order to operationalize this query in our questionnaire, we further identified in the literature what were the common themes in recent library building projects.

\section{Themes in contemporary library building projects}

The review of the literature revealed a fairly consistent set of themes in recent library building projects, especially technology, hours/access, study areas, aesthetics, and amenities. Many studies identified technology improvements, especially additional workstations and outlets for laptops. Thus, Holder and Lange (2014) found students at their library requested additional outlets for laptops and a variety of furniture for study areas. Halling and Carrigan (2012) compared seventeen space-change options and found that seating, rooms/booths/carrels, group tables, quiet study spaces, computers, and power were the most commonly requested at the Medical Sciences Library at Texas A \& M University. In addition to technology, researchers also identified the need for more and more diverse study spaces. For example, Bryant, Matthews, and Walton (2009) identified diverse study spaces, intrusions and interruptions, technology, diversity, library staff and materials, and spatial organization as the key themes in their case study of the Loughborough University Library's implementation of a learning commons area. Bailin (2011) found that collaborative/individual study, spatial design, social space, technology, noise levels, and access to help were the most important at the University of New South Wales Library. Applegate (2009) identified capacity for the end of the semester "surge," study spaces, wireless internet, power for laptops, and accommodation of both men and women patrons as key design determinants. Apart from study spaces specifically, studies also revealed a need for furnishings and amenities that promote a more comfortable, social atmosphere. Montgomery (2014) found tables (e.g. for group projects), outlets, comfortable furniture, a café, computers, and quiet ambience as the improvements most requested by the library's user-group. Latimer (2010) identifies as key design trends in art libraries: exhibition space, varied study and seating areas, separation of social and quiet spaces, customized shelving, access to e-resources, merging of help desks (library reference, IT, etc.), clear signage, 24-hour access, good lighting, and quality design. In the renovation of the Auraria Library Brown-Sica (2012) found patrons requested more computers, more/better furniture, a more attractive environment, and electrical outlets most of all, and in consultation with other stakeholders, Somerville and Brown-Sica (2011) report the development of the five areas of study areas, programmatic areas, amenities, operations (e.g. facilitating library workflows), and building (e.g. environmental control) as the priorities for that renovation. Lawrence and Weber (2012) add to a growing literature that demonstrates that patrons of academic libraries want 
and will use library spaces and services beyond conventional hours, especially in the period from midnight to 2:00 a.m. Other researchers found user preferences in many of these areas, which is also true for many of the studies noted above. Thus, Fox and Doshi (2013) found that a renovation of a library study area according to the themes of power/data (for laptops), lighting, aesthetics, and a "defined yet open" scheme dramatically improved the use and user satisfaction with the space. Vaska, Chan, and Powelson (2009) found that the most-requested improvements at the Health Sciences Library at the University of Calgary were more electrical outlets, study carrels with lighting and outlets, study/meeting rooms, and recliner-type chairs. Ludwig (2010) identified cafés, small group study spaces, large group training spaces, wireless access, temporary work spaces, and quiet places as key themes in medical library design. From this voluminous and diverse literature, we identified as core themes to be investigated in this survey technology, hours/access, study areas, aesthetics, and amenities. Less central themes, such as spatial design, variable capacity, signage, operations, and accommodations for gender and ethnic preferences were omitted from this survey, though continue to be of interest for future research; open-ended questions in the questionnaire would help identify areas of interest outside the core group.

\section{Space and culture}

The final major theme we identified in the literature review was that many academic libraries were being re-imagined as social spaces that responded to, accommodated, or even inculcated a particular culture among their patrons. As academic culture has changed to include more social and group-oriented study, libraries have seen the need to develop themselves as social spaces, but finding the balance and the effect on traditional services remains controversial. Stewart (2010) contrasts academic library buildings from mid-century to the 1980 s, characterized primarily as utilitarian, with an emerging trend that uses the library building to communicate the values of academic culture, especially involvement in a community of learners. This trend includes the reimagining of the library as a learning commons designed to support a shift in pedagogy toward collaboration and group projects (Yoo-Lee, Lee, and Velez, 2013), but also the inclusion of cafés and other spaces not targeted specifically at learning, but primarily at enhancing the social element of the space. Demas (2005) argues for a conceptual model that accommodates both academic activity and social activities such as socializing, eating and drinking, and participating in cultural events. Many studies (Yoo-Lee, Lee, and Velez, 2013; Bennett, 2007; Montgomery, 2014) report that individual users found social spaces conducive to work because of the ambient noise, the presence of others working, or similar reasons. Bennett (2007) makes the case that the social aspect of learning spaces on university campuses, such as libraries, is one of the key rationales for continuing to invest in brick-and-mortar facilities. On the other hand the literature shows a controversy about the long-term costs and benefits of reimagining the library as a social space. 
Gayton (2008) argues that the "communal" experience of academic libraries (i.e. individual study in the presence of others) is more central to their core function, and the social experience of libraries should not infringe on it. Lippincott (2010) notes that introducing learning commons can be counterproductive if the project is not imagined from the point of view of the mission or vision of the library. Other researchers describe the need to fit the culture of their individual library with the broader institutional culture. Montgomery (2014) describes renovations at the Olin Library at Rollins College as supporting an institutionally determined learning culture (the “Conference Plan"). Webb, Schaller, and Hunley (2008) found that their library's design encouraged individual study contrary to the collaborative emphasis of their university context, and planned to redesign the library to align it with the university culture. Thus, while the reinvention of the library as a social space responds to real needs in the changing academic context and many libraries are building cafés and lounges, the literature also voices concerns over these changes, and suggests that such projects should take the particular institutional context of the library into account.

\section{Research questions}

The trends we identified in the literature sometimes agreed with the intuition of the library faculty and requests from individuals at the university, but also sometimes raised questions about how these trends might appear in our particular context. Thus, while the literature suggested that print collections had diminishing importance for undergraduates, anecdotally, the faculty librarians at Whitworth Library sensed that while their patrons tended to prefer electronic formats for academic journals and other periodicals, they still preferred print formats for monographs and reference materials, and often still searched by browsing the print collections. Therefore, we wished to confirm what our user-group in fact preferred and expand our knowledge of their preferred formats for other item types, so that we could arrange our spaces to provide the easiest access to the most preferred formats. Therefore our first research question is: What formats do our user-groups prefer for the content types provided by the library?

Informally, students had requested extending library hours and providing a 24-hour room, and library faculty also recognized that the current library hours were not sufficient for students' needs. The literature confirmed that these were widespread trends in expanding academic libraries and library services, but we wanted more information on our user group's specific needs for study space before embarking on renovating or re-purposing library space. The literature review also identified as important trends improving technology (especially more computers, better wireless internet, and more electric outlets), providing diverse study areas, improving aesthetics (especially lighting, furniture, and shelving), and amenities (especially food and drink), which were also widely identified as user needs. Since few of these studies surveyed 
populations similar to our own user group, we wanted to know how our patrons ranked these improvements and whether they identified further needs. Therefore our second research question is: What aspects of library spaces do our patrons identify as most important, either expanding/improving existing spaces or introducing new spaces?

Prior to undertaking the survey the library faculty and other members of the university community had expressed interest in expanding amenities in the library that would increase the functionality of the facility as a social space and contribute to its role as symbolizing the values of intellectual curiosity and the free exchange of ideas. Our review of the literature confirmed that similar projects had been undertaken by other libraries and provided a theoretical justification for the social function of the library space. It remained to query our user group about its preferences and priorities for such improvements. Proposals were made for a café (with several levels of service considered), a lounge (sofas, fireplace, etc.), and an event venue for book signings, guest lectures and the like. We wanted to see if our user-group would make use of such spaces, and if other social or cultural spaces were desired. Therefore, our third research question is: What social or cultural spaces in the library do our patrons identify as ones they would use?

\section{Method}

A questionnaire of thirty questions was distributed and managed through online survey software (Qualtrics). Questions were divided into three basic categories: how students currently use the library space and collections, what personal benefit students perceive from proposed changes, and what students would like to see improved in the library. All questions were optional, and some questions were clarifying questions presented to the student based on their previous responses.

\section{Site: Whitworth University}

Whitworth University is a small liberal arts college on a walk-able, pine tree covered, 200-acre campus. Students and faculty refer to the campus as being behind the "pine cone curtain," communicating the retreat-like feel of the open forest and brick building campus, and the close-knit community of faculty, staff, and students. Whitworth highly values an engaged campus community as an opportunity for learning, mentoring, and intellectual inquiry. To foster that community, all students are required to live on campus for the first two years of college after high school graduation (exceptions are made in some circumstances). In spring semester, 2015, 49\% of all students lived either on-campus or in campus-related housing (G. Whisenand, Director of Institutional Research, personal communication, 16 April 2015). The library is situated in the center of the campus, making the library easily accessible for most students. Total enrollment for spring semester, 2015 was 2878, 2560 of whom were 
undergraduates and 318 were graduate students. The majority (87\%) of undergraduate students are "traditional" day students. The remaining $13 \%$ are in the Continuing Studies program: students who take classes evenings and weekends either on the main Whitworth campus or at a downtown center approximately seven miles from the main campus (Whitworth University Institutional Research, 2015).

\section{The questionnaire}

Most questions on the questionnaire were adapted from surveys published in the literature (Brown-Sica, 2012; May and Swabey, 2015; Fox and Doshi, 2013; American Association of Law Libraries ALL-SIS Student Services Committee, 2011); we also created new questions that would help us assess our user-group. In particular, questions on activities performed in the library, the importance of collections and spaces to assignments, frequency and purpose of visits to the library, and technology needs were adapted from surveys published in the literature. Since specific suggestions for a café, extended hours, and a 24-hour study room had been made from within the campus community, we created questions to assess these directly (hours and cafés were discussed in the literature, but specific survey questions were not published). A few unrelated questions on topics for library instruction and signage were added on the request of library faculty. Two drafts were tested by eighteen student library employees. While this group was not representative of the larger student body because of their clearer understanding of the functioning of the library, these first tests helped clarify question syntax and indicated whether or not the questionnaire was too long. On completing the draft survey, students emailed written evaluations on clarity of the questions and suitable length of the survey. These students were motivated by our request for feedback to complete the entire survey, so survey completion times recorded by Qualtrics were also used to determine whether or not the survey was of an appropriate length. The number of questions was not large; however, the almost equal distribution among multiple choice, Likert-type, and open-ended questions made the completion time longer than might be expected from a thirtyquestion survey.

Beyond fine-tuning terminology and wording, some questions were combined or eliminated to reduce completion time. The questionnaire structure was re-organized to place questions about current and potential building use at the beginning of the survey so that data could be gathered more comprehensively even in cases of survey drop-out. A third draft was tested by sixteen student writing tutors. Their input was quite valuable: their training in composition and more general understanding of the library helped further refine questions into a questionnaire that did not assume an understanding of library functions and was understandable to the wider student population. A fourth and final draft was developed based on written evaluations from the student writing tutors. The final draft retained all 30 questions 
of the original, but some open-ended questions were converted to multiple-choice, multipleselection questions, and six questions were made contingent on specific responses. The revisions primarily addressed understandability and the need to keep the time-commitment from becoming onerous.

\section{Data collection}

The target population for the survey was all Whitworth students enrolled in spring semester, 2015: on- and off-campus, undergraduate and graduate, and traditional and nontraditional. The final questionnaire was distributed to the entire student body via email through the Associated Students of Whitworth University (ASWU). No incentives to complete the survey were offered. The email from ASWU was sent at 3:43 p.m. on a Thursday afternoon, and the majority of completed responses was received within twelve hours of the email. Other methods of distribution included a paid ad on the library's Facebook page with a link to the survey and setting up a table outside the campus cafeteria during the lunch hour for two days. During that time, library staff had two iPads available for students to take the survey and also distributed small flyers with a printed link to the survey. While the other efforts at increasing student participation were moderately valuable in getting more students to respond, the best and largest response came within the twenty-four hours after the email was sent from ASWU (Figure 1).

[insert Figure_1.jpg]

Figure 1. Survey response dates.

The survey was kept open for ten days. A total of 497 students responded. Of those responses, twenty-six completed only $5 \%$ of the survey, responding only to first two questions (academic year and major). Those responses were discarded due to the insufficient data, leaving a total of 472 responses in the final data set, or $16 \%$ of the total target population. All other partially complete surveys were included in the final data set; total number of respondents $(n)$ for each question will be noted. Further processing of the raw data consisted of coding multiple choice and Likert-type questions (done automatically by the survey software) and attribute classification of open-ended questions.

\section{Results}

The respondents to the questionnaire closely matched the target population in year of study (Table 1) and field of study (Figure 2). Five of Whitworth's largest majors had the highest response rates.

[insert Table_1.jpg] 
Table 1. Survey respondents' academic status compared to Whitworth University Spring 2015 enrollment.

[insert Figure_2.jpg]

Figure 2. Top majors of respondents compared to top majors at Whitworth, Spring 2015.

\section{Use of current library space}

Most respondents report using the library often: $76 \%(n=459)$ of respondents come to the library at least weekly (Table 2). Their most common tasks in the library are to study on their own, use the printers, study/work in groups, use the computer labs, use print materials, and use scanners/copiers (responses chosen by over $50 \%$ of respondents, listed with highest response first). Students who visit the library less frequently (monthly or a few times a semester) report doing these same tasks but with a different order of preference: printers, study/work in groups, study on own, use the computer labs, use print materials (responses chosen by over $50 \%$ of respondents, listed with highest response first). For both groups, spaces for study, access to equipment, and access to print resources are important services the library provides.

[insert Table_2.jpg]

Table 2. Ranking of library tasks based on frequency of library visits.

Information behavior and format preferences

The questionnaire divided questions about information behavior into two parts: how respondents actually used available resources and which ones they considered important for their academic work. Despite primarily making use of digital discovery tools, our user group indicated a preference for print books, and a significant number used the physical book stacks for resource discovery. Electronic databases were the most used discovery tool. With a mean value of 3.82 ( $n=318$ ) on a 1-5 scale (5 indicating the highest use), article databases are used most frequently by students. The book catalog is also used frequently by students (mean value of $3.19, n=319$ ), but the majority of the resources discoverable there are print ( $47 \%$ e-books, $53 \%$ print books; the catalog also includes records for print journals, CDs, and DVDs/videos, but not e-journals, streaming music, or streaming video). Over half of respondents $(52 \%, n=308)$ browse the physical stacks to locate resources sometimes, often, or all the time (Figure 3 ).

When asked about how important different formats are for their academic work, students demonstrated a preference for print monographs and digital journals over e-books and print journals (Figure 4). The need for print-format resources also brings users into the library: $60 \%$ of respondents $(n=467)$ report coming to the library to use print resources, with 
$21 \%(n=467)$ coming to the library specifically to browse the print collection (Figure 5$)$. These results confirmed what librarians had surmised: that our user group prefers digital formats for discovery and use of journal articles and for some discovery of print books, but physical books remain important for discovery and use.

[insert Figure_3.jpg]

Figure 3. How often do you use each resource?

[insert Figure_4.jpg]

Figure 4. How important is each collection for your academic work?

[insert Figure_5.jpg]

Figure 5 . Tasks in library categorized by type.

\section{Functional needs}

The survey also asked respondents to evaluate potential improvements to the library's physical space. Our users identified two themes as very important: equipment and study space. Many desired improvements derive from the building design from 1992 that did not anticipate the nearly universal use of multiple personal electronics for study (laptops, smart phones, and tablets). Throughout the questionnaire, respondents requested better wireless access. Some students took the opportunity to ask for better wireless access in every open-ended question, related or not. When asked what the library needs more of/needs to improve, $70 \%$ ( $n=267)$ indicated "improved Wi-Fi" (11 options were given, respondents could select multiple responses). Asked what equipment the library needs more of (open-ended), 34\% ( $n=152$ ) said "better Wi-Fi" or the like. Other requests similarly relate to the dated building design: respondents did not like fluorescent lighting, would like more large tables to spread out books and electronics, and would like more outlets for charging devices. In an open-ended question asking how to improve library space in general, $26 \%(n=237)$ requested better lighting, with $20 \%$ of those respondents specifically mentioning an extreme dislike of the fluorescent lighting. One student stated. "The lighting is the one thing that is keeping me from the library because the lights strain my eyes."

Study spaces. Respondents overwhelmingly requested a 24 -hour study space $(78 \%, n=440)$, with access to printing, comfortable chairs, Wi-Fi, and food/coffee. While the majority of respondents listed equipment and physical study space as the most important to a 24-hour area, a significant number desired access to library-specific functions: print library materials (40\%), reference/research help (16\%), and circulation (14\%).

Current use of the library and requested improvements primarily involved study space, both for individuals and groups. Students were asked what they do in the library and given 
nineteen possible activities, and their second and third highest responses were to study individually and to study in a group (Table 4). Similarly, the top five facilities/services requested within a 24-hour study space were (in order of preference): printers, individual study space, Wi$\mathrm{Fi}$, food/drink, and group study space. In a multiple-choice, multiple-response question students were asked what the library needs more of and were given eleven options to choose from. Group study space came second only to improved Wi-Fi for respondents when asked what space/facilities the library needs to improve. Less important to respondents were more scanners and more computers (see Table 3 ).

[insert Table_3.jpg]

Table 3. Services/facilities requested in a 24-hour space.

An unanticipated need for quiet study space came up throughout the questionnaire: in questions on a proposed 24-hour study space, a proposed coffee chop, what equipment the library needs to expand, and what furniture the library needs more of. Several respondents objected to the proposed coffee shop on the grounds of noise alone. Students noted that there are other spaces on campus for study with ambient noise, such as the coffee shop, dorms, and other academic buildings. Respondents who answered affirmatively that the library needed more individual and group study space were asked further clarifying questions about those spaces. Most students who desired more individual study space wanted quiet space $(85 \%$, $n=209)$, not low noise $(13 \%, n=209)$. A narrower margin of respondents preferred quiet group study space: $61 \%(n=232)$ preferred quiet and separate (closed rooms), with $37 \%(n=232)$ preferring low noise and common space (Table 4).

[insert Table_4.jpg]

Table 4. Type of individual and group study spaces requested.

In other indications of our user-group spending considerable time in the library, $32 \%$ $(n=237)$ would like more comfortable chairs and/or couches for long-term studying. Another interesting suggestion to accommodate long-term studying was for standing desks.

Hours. Survey data indicate that students often use, and want to use, the library outside of the traditional 8-5 workweek hours. The exception to that trend is mornings: the majority of respondents $(76 \%, n=405)$ would never or rarely use the library before 7:30am weekdays or before 9am Saturday $(69 \%, n=410)$. Many students would sometimes or always use the library after midnight on weekdays $(57 \%, n=423)$, but most would prefer later hours on Fridays (64\%, $n=422$ ) and Saturdays $(69 \%, n=428)$ (current library closing times are 5:30 p.m. and 6:00 p.m., respectively). 
Results from questions about use of the physical library space indicate our user group would like more and better: more of the types of currently available study spaces, longer open hours, and improved quality of equipment.

\section{Social and cultural needs}

Survey questions did not explicitly ask about respondents' perception of the library and campus culture. Rather, insights into the social needs of respondents and campus culture came in two ways: first through open-ended questions regarding current use of the library space and desired improvements, and second, in a series of questions, four types of spaces were proposed for evaluation: café/coffee shop, an event venue, a study lounge, and presentation rehearsal space.

Respondents want to have food and drinks while studying, but they do not necessarily want a coffee shop. A slightly higher number report they would use a study lounge more often than a coffee shop. Asked how often they would use a coffee shop in the library, $66 \%$ ( $n=398$ ) respondents reported they would use it once a week or more. Similarly, $71 \%(n=290)$ of respondents would use a study lounge (defined as a quiet, informal space with comfortable seating and a fireplace) once a week or more. Open-ended questions also indicate that students want a quiet, comfortable area to study where they are free to have coffee and snacks. Some students rejected the idea of a coffee shop outright, noting in open-ended questions that that type of space is already available on campus. What the library provides, and what they can't find elsewhere on campus, is a quiet place to study that has access to printers, scanners, computers, and research materials. Students mentioned that the only 24-hour study spaces on campus are the dorm lounges, which are neither quiet nor do they have the equipment students need for study. Although most respondents expressed positive interest in a coffee shop and in a lounge, students expressed moderate interest in a presentation rehearsal space and in an event venue (defined as a space for faculty/guest lectures, book readings, etc.). A majority of students would use these spaces only a few times a semester, but that is probably the expected use of such spaces.

Responding to questions regarding the atmosphere and furniture in the library, some phrases that came up repeatedly in open-ended questions were "more comfy," "more cozy," and "warmer colors." Students also mentioned wanting an atmosphere similar to the coffee shop on campus. Students commented that "The coffee shop in the HUB is always so packed" and "the [coffee shop in the] HUB is always full." The HUB (Hixson Union Building) coffee shop was remodeled in recent years and it has all of the components requested by students for the library space: comfortable chairs, warmer color scheme, electrical outlets, functional Wi-Fi, and coffee and food. Taken with the responses to the proposed library spaces, our users do not 
need more social space in the library, but rather would like to combine the atmosphere of a coffee shop with library study space.

\section{Discussion}

The results of the survey were helpful in clarifying the needs of our patrons and prioritizing future renovations; at times they were in alignment with trends identified in the literature, but at other times they identified needs particular to our community and indicated areas that needed further explanation. Based on the results of the survey, we identify six foci of particular interest to our user-group: spaces, technology, hours/access, supporting study, aesthetics, and amenities.

Spaces (collections vs. common areas)

The literature review established that, given changes in format and space needs, many libraries confront the option of converting shelving space to support a stronger learning commons focus in the library (Burton and Kattau, 2013; Vaska, Chan and Powelson, 2009). While acknowledging the value of such a space, the library faculty also had the impression that many of our patrons still prefer print format for books and search by browsing. The survey helped address the tension between these two points of view and confirmed that our users identified print books as important for their academic work third after electronic indexes/databases and electronic journals. A significant minority indicated that they browsed the print collection "often" to find resources, and some came to the library for that purpose. This was not surprising, especially given the importance in our collections, curriculum, and campus culture of the disciplines of theology and education, which tend to favor print formats. Conversely, our patrons indicated that electronic books were a less-preferred format. Several factors seem to contribute to that preference. Again, availability of key resources in important subjects in our collection is one, but, also, the majority of our electronic book collection is a bundle, not individually selected by the faculty and librarians as our print collections are. Further, there is the significant minority who prefer to search by browsing. Our patrons ranked other parts of the print collections, journals and indexes, as being less important for their academic work. In making additional space available for study and productivity, these appear to be the best candidates for consolidation or storage.

\section{Technology}

The technological needs identified by our user-group focus on technologies that support their personal items, such as wireless access and wireless printing, with fewer responses calling for expansion of computer labs or workstations in the library. Although slightly more respondents reported using the computers in the library than using their laptops, when we asked about improvements on existing spaces, improved wireless access was the most common 
request, with additional computers and computer labs at the bottom of the list. In addition to selecting improved wireless access as a key improvement, many respondents reiterated this need in the open-ended question on improvements, with comments such as "seriously, improve the wifi!!!". No responses in the open-ended question specifically called for more workstations, but several indicated an interest in shifting the balance from the labs to the study areas. Similarly, respondents ranked the need for more scanners as low, and individual comments such as "photo scanners for reserve books" suggest that a rearrangement of the current equipment to make it more convenient would satisfy our patrons' needs. Interestingly, respondents placed wireless printing high on the list of improvements, despite the fact that the library introduced wireless printing about six months prior to the survey. Comments such as "we have wireless printing?" suggest this is as much a matter of communicating this service to our users as actually improving its functionality. A moderate number of respondents asked for more electrical outlets for their laptops or USB sockets for their tablets. This ran counter to what we had seen in the literature, where these were often among the highest demands, suggesting that our building is relatively well supplied with these for the needs of our usergroup and that this is a lower priority. A few respondents indicated in the open-ended response the need for specific software, but the paucity of such feedback suggests that the current software available is a good fit for our patrons and not a high priority for expansion.

\section{Hours/access}

The campus community makes consistent use of the library building, with the majority of respondents reporting that they visit the library either 2-3 times a week or daily. Our usergroup confirmed broader trends showing that students expect library facilities to be available at their convenience, $24 / 7$ if possible. Our patrons indicated a preference for longer hours Friday and Saturday evenings, which seems particular to our user-group, as, e.g., Lawrence and Weber (2012) found the highest demand for late-night hours Sunday through Tuesday. This may reflect the relatively high proportion of residential students in our user-group; they are not making a special trip to campus to use the library, but use it as part of their regular study routine. As the university plans to expand offerings in continuing education and graduate programs, the need for off-hours access will likely continue to increase. Although we did not include an open-ended question about hours, a few respondents indicated elsewhere in the questionnaire the need for the library to be open Sunday mornings also, but this is currently not a viable option because of the institutional commitment to reserve this time for worship services. As with many libraries, expanding hours calls for a significant investment, particularly in expanding staff, but one that our library needs to consider seriously since there is a clear preference among our users for extending evening and late-night hours. 
The option of establishing a 24-hour study space in the library is clearly one that our patrons support. It could alleviate some of the pressure to expand hours without having to adjust the library staff. Respondents overwhelmingly indicated that they would use a 24-hour study space if it were available. In the open-ended question about the space, many students indicated strong support with comments such as "This would be incredibly helpful as a safe, quiet place to study on weekends in particular when it is hard to find somewhere to go. Nighttime is also hard on campus on weekdays when everything shuts down yet there is still work to do." The library was the most preferred location on campus for such a space, which supports the general sense that our user-group associates the library with study and productivity, and that there is a lack of other quiet, study-friendly areas on campus. The relationship of such a space to the library needs to be carefully considered; this was an important finding of our research as we did not find the issues regarding the relation of 24-hour study spaces and the library discussed in the literature. On the one hand the most requested services (printers, individual study spaces, wireless access, food/drink, and group study/work spaces) could be separate from the existing facilities, but others also requested library-specific services (access to print materials, reference/research help, and circulation).

\section{Supporting study}

Many survey questions asked students to evaluate various aspects of the study areas in the library. In particular, open-ended responses were helpful for us to get a broader picture of the campus culture regarding study space. Students mentioned using the residence hall lounges, as well as the coffee shops in the student union building and science building as other study spaces on campus. All of these spaces provide comfortable seating, wireless access, and varying degrees of ambient noise (although, anecdotally, "ambient" does not describe the noise in typical residence hall lounges). Survey responses suggest that in the larger picture of the campus, the library occupies a unique niche as a place for quiet study, research, and group work. Considering both how respondents currently use the library and what they would like to see more of, our user-group demonstrates a strong preference for productivity tools (computers, printers, and scanners), group workspace, and individual quiet study space. These three items consistently appear in the top responses to several questions ranging from "what do you do in the library" to "what facilities/services would you want to use in a 24-hour study space" to "what spaces/facilities does the library need more of."

More particularly, of those who responded that the library needs more group spaces, and those requesting more individual study spaces, both requested quiet, separate spaces rather than low noise and common spaces. The learning commons model, which is often defined as a place for social activity and study (both collaborative and individual) with ambient noise, does not fit the needs and expressed wishes of our particular user-group. Since those 
types of areas are available elsewhere on campus, the library should embrace its reputation for quiet and provide an alternative to the busy, highly social, multi-purpose study areas on campus. This enhances campus culture by providing a different type of study space rather than replicating ones already available. As one respondent writes, "I also worry that if we make the library into more of a hangout place it will get noisy. $A[n y]$ noise at all destroys my concentration, and the library is one of the only places on campus to go where it is reasonable to expect quiet." Our findings here may, again, relate to our specific user-group as being largely residential students on a small campus; for these users the library does not need to replicate spaces that are available nearby, in contrast to, e.g. the Auraria Library's need to provide social spaces for a large commuter-student population which does not otherwise have access to such social spaces (Brown-Sica, 2012). Here our findings broadly support Gayton's (2008: 60) distinction between "social" and "communal" experiences of the library, where he sees current trends to introduce social spaces as undermining the traditional communal nature of the academic library, defined as the "experience of seeing and being seen by others, quietly engaged in the same serious, studious activity."

The expressed needs of our user-group reflect the broad trend toward instruction using collaborative group projects found in the literature review (Yoo-Lee, Lee, and Velez, 2013). Group study rooms were ranked as highly used in the current building and as the most desired facilities in a 24-hour space. For areas in the library that needed improvement, group workspace was second only to improved wifi. However, the corresponding requests for quiet space (both for individual and group study) indicate that the more social, communal model of the learning commons may not meet the group study needs of our user-group. Bennett's (2007: 17) research made distinctions between "quiet" and "free from distraction." This is an important distinction, especially when interpreting our students' seemingly antipodal request for quiet group study space.

\section{Aesthetics}

Our patrons conformed to the general trend that library users expect their spaces to be comfortable and inviting, not merely utilitarian. The most noted outdated design element in the library was the lighting. The early 1990s fluorescent lighting was never meant to support the lighting needs of students with multiple computing screens, and respondents reasonably requested that it be reduced or eliminated. Respondents made a variety of suggestions including more natural light, lower light, and more individual desk lamps. However, given the extreme dislike of fluorescent lighting expressed in the survey, eliminating the fluorescent lighting would likely be satisfactory to all in our user-group no matter what type of nonfluorescent lighting replaces it. Citing library use for long-term study, respondents also asked for more comfortable chairs, both to add lounge-type chairs and to replace the existing partially 
wood table chairs. Other potential updates to the 1992 design include warming up the color scheme and adding larger tables to spread out with study materials and laptops. One respondent wrote that with the lighting, uncomfortable chairs, and 1990s blue-and-maroon color scheme, the library is "not a place you would want to sit at for periods of time." And yet many students do, owing to the lack of other adequate space on campus. Both the need for more areas for quiet, focused study and the need to update the aesthetics indicate a larger need to create a building-wide atmosphere for long-term study. Noise level, furniture, lighting, and wall color all contribute to the overall atmosphere of the library.

\section{Amenities}

We were surprised by the mixed response to the idea of including a café in the library, given that this idea was welcomed by other user-groups as reported in the literature. On the one hand the majority of respondents indicated that they would use a café if it was in the library at least once a week $(66 \%, n=262)$, though in the open-ended question asking for general input on the library space, individual students were strongly opposed to the idea, noting that "That area would be crowded and loud and the[re] would be a noticeable difference in the usage of the library. I like keeping my library separate from by food areas personally. Plus, both campus coffee shops are super close to the library as it is," and "There should not be a coffee shop in the library. I enjoy the library as a quiet study space." Other individual respondents were in favor of the idea, "A coffee shop would be fantastic!" but the general response was more ambivalent than seen elsewhere in the literature. This supports the warning of Waxman, et al., (2007) about mediating noise issues arising from library cafés. Respondents more univocally supported the idea of a lounge area in the library (described in the questionnaire as "comfortable seating, fireplace, informal meeting, casual reading, etc."). This was the highest preference for a new space in the library, the majority of respondents indicating they would use it at least 2-3 times a week $(53 \%, n=205)$. Individual responses to the open-ended questions on new spaces and general input suggested this space would contribute to the library as a space for individual inquiry and discussion, as one respondent wrote, "comfortable lounge chairs or couches for hours of reading", and another wrote, "devote a space to more informal studying, i.e., couches, fireplace, in general a more relaxed/less rigid atmosphere." We also asked about including an event venue for book readings/signings, guest lectures, and the like, but most respondents indicated they would use this a few times a semester or never $(65 \%, n=251)$. Those responses partly reflect the nature of such a space, given that it would probably not be feasible to schedule events weekly. On the other hand, no respondents indicated any interest in such a space in the open-ended question about new spaces, so the development of such a space is evidently a low priority.

Strengths, limitations, and areas for further research 
In terms of an exploratory survey probing the information behavior and perceived needs of our user-group for the library facilities, the primary strength of this study is that its target population was the student body of Whitworth University as a whole. The questionnaire was open to the whole student body, and was not distributed or administered in the library, but distributed by ASWU. Wakaruk (2009) argues for the importance of studying the needs of both library users and non-users. We had a response rate of $17 \%$ ( $16 \%$ once incomplete responses were removed). Since participants self-selected, there may be some selection bias toward students who are already library-users, but, given our response rate and the observation that the distribution of respondent's majors closely matched that of the student body as a whole, we are confident that the respondents are highly representative of the overall target population.

The results of this survey are limited in that they are directly applicable only to our target population, and cannot be universalized to other user-groups. As discussed above, the library-space needs of our users seem to be influenced by the size and layout of this particular campus, distribution of academic disciplines, and a more intangible cultural element. Since this was an exploratory survey, we could not draw firm conclusions about the exact nature of those influences, but this remains an area for future research. Second, the survey was limited in that we employed only the single questionnaire instrument; while we had a good response rate, other instruments such as observation would help confirm the correlation between how respondents report they use the library space and how they in fact use that space.

In addition to local influences on use of the library space, the survey suggested further areas for future research. We suspect that our patrons are in a period of transition in terms of their information behavior and preferred formats and environments. Tracking longitudinal data on these preferences will help map the nature of that transition and clarify other influences, such as academic discipline and local culture. Collecting longitudinal data as we proceed with improvements is another important project in clarifying how specific improvements affect the use of library space and the perceptions of the affected user-group. While many studies report data collected before or after such projects, few provide comparisons with "before" and "after" (a notable exception is Fox and Doshi (2013)). Our study also indicated that the role of social spaces in the academic library is still largely terra incognita; some studies have indicated very positive results from introducing such spaces, but the long-term effects of such spaces on the perceived role of the academic library and its cultural contribution are still unclear. This was reflected in our respondents' ambivalence about such spaces, and remains an area for continued research.

\section{Conclusion}


As an exploratory survey of our user-group, this study was successful in identifying the unique characteristics of our patrons, and in helping prioritize how we should proceed in improving the library facilities. Especially important were the results that showed our users' behaviors and preferences ran counter to the prevailing trends identified in the literature. Where the literature emphasized the growing importance of digital formats and the diminishing importance of print, our users tend to be conservative in their format and information-seeking preferences; while most prefer electronic databases and journals for finding and accessing journal literature, they prefer print format for books, and a small but significant group prefers to browse the physical stacks. Further research might show that these preferences are split along disciplinary lines, which could help us decide where physical holdings could be converted to electronic holdings. At present our patrons would not support a wholesale conversion of physical shelving to make room for an expanding learning commons.

Many of our patrons use their own devices (laptops, tablets, smart-phones, etc.) for research in the library, so the greatest technology needs are in supporting those. This is again an important refinement from the dominant image in the literature, which seemed to indicate that university students demanded major investments in technology of all kinds. In contrast, our patrons identified clear priorities in this area as improving wireless access and making wireless printing more visible or easier to use; there was not a large call for a new common space with many new computer workstations in the learning commons style. Our user-group was in line with current trends when it came to hours and access, requesting the longest possible hours, with a particular preference for longer evening and late-night open hours. They showed strong support for the idea of a 24-hour study space associated with the library, supporting the general impression that they associate the library with study and productivity. Expanding hours, especially on Friday and Saturday evenings and introducing a 24-hour study space are also high priorities. This again helps refine the trend reported in the literature, especially as it seems likely we can connect the preference for Friday and Saturday night hours with the specific context of Whitworth Library as being on a small residential campus, a type generally under-represented in the literature.

Our patrons consistently requested more study space, both individual study spaces and group work and study spaces, but also indicated a strong preference for zoning between strict quiet areas and work areas. Few respondents supported a common, more social area, typical of the learning commons model, despite the wide support for this trend in the literature. Our user-group's response to the aesthetics of the library space confirmed broader trends in that they see the library as a cultural space, not simply a functional repository. Updates and routine maintenance in lighting, paint, and furniture that help construct an atmosphere that communicates a culture of intellectual inquiry, collaboration, and creativity are a priority for our patrons. Many patrons also supported a café-space within the library, but such an 
expansion should be approached carefully so as to meet concerns about noise from such a space disturbing the rest of the building; this importantly substantiates earlier intuitive warnings (Waxman, et al, 2007), identifies an important factor arising from our unique context, and contributes to the ongoing debate over the relative merits of communal and social spaces in the library. Finally, our users indicated a strong preference for a lounge-like area that would support casual reading, informal meetings, and, again, support a culture of communication and collaboration.

Our study confirmed for us the importance of balancing trends in the literature with direct investigation of our patrons; as Martin (1976: 495) once admonished "eliciting user response should be an integral part of the ongoing practice of the librarian." While the literature was invaluable in identifying the broad areas that needed to be considered in assessing our space needs, it was also necessary to employ the experience and intuition of the library faculty and the university community as a whole to develop the research and identify the best ways to meet our users' needs. As university instruction, technology, and campus culture continue to evolve, we plan to continue surveying our patrons both to gather longitudinal data on these trends and to evaluate the efficacy of our responses to them.

More concretely, as a result of our research, the library director has implemented several changes to library spaces and amenities, including offering free coffee in the library during finals week, placing baskets of earplugs throughout the library, purchasing portable standing desks for library use, and making more private rooms available during final exams. An investigation is being undertaken into the causes and solutions for problems with wireless access in coordination with the university IT department. Finally, a large, enclosed room that had been used for current periodicals, and more recently for a large exhibit has been converted to a comfortable quiet study space. Updates to the space include a gas fireplace, warm lighting, and a variety of comfortable chairs and tables. 


\section{Works Cited}

American Association of Law Libraries ALL-SIS Student Services Committee (2011) Model Student Satisfaction Survey. Available at:

www.aallnet.org/sections/all/storage/committees/students/studentsurveyssubcmte/m odelsurveyallsis-final.pdf (accessed 15 December 2014).

Applegate R (2009) The library is for studying: student preferences for study space. The Journal of Academic Librarianship 35(4): 341-346.

Bailin K (2011) Changes in academic library space: a case study at the University of New South Wales. Australian Academic \& Research Libraries 42(4): 342-359.

Bennett S (2007) First questions for designing higher education learning spaces. The Journal of Academic Librarianship 33(1): 14-26.

Black C and Roberts S (2006) Learning the social way: enhancing learning in a traditional setting. New Review of Academic Librarianship 12(2): 83-93.

Brown-Sica MS (2012) Library spaces for urban, diverse commuter students: a participatory action research project. College \& Research Libraries 73(3): 217-231.

Bryant J, Matthews G and Walton G (2009) Academic libraries and social and learning spaces: a case study of Loughborough University Library, UK. Journal of Librarianship and Information Science 41(1): 7-18.

Burton F and Kattau M (2013) Out of sight but not lost to view: Macquarie University Library's stored print collection. Australian Academic \& Research Libraries 44(2): 102-112.

DeClercq CP and Cranz G (2014) Moving beyond seating-centered learning environments: opportunities and challenges identified in a POE of a campus library. The Journal of Academic Librarianship 40(6): 574-584.

Fox R and Doshi A (2013) Longitudinal assessment of "user-driven" library commons space. Evidence Based Library and Information Practice 8(2): 85-95.

Gayton JT (2008) Academic libraries: "social" or "communal?" The nature and future of academic libraries. The Journal of Academic Librarianship 34(1): 60-66.

Given LM and Leckie GJ (2003) "Sweeping" the library: mapping the social activity space of the public library. Library and Information Science Research 25: 365-385. 
Halling DT and Carrigan E (2012) Navigating user feedback channels to chart an evidence based course for library redesign. Evidence Base Library and Information Practice 7(1): 70-81.

Holder S and Lange J (2014) Looking and listening: a mixed-methods study of space use and user satisfaction. Evidence Based Library and Information Practice 9(3): 4-27.

Latimer K (2010) Redefining the library: current trends in library design. Art Libraries Journal 35(1): 28-34.

Latimer K (2011) Collections to connections: changing spaces and new challenges in academic library buildings. Library Trends 60(1): 112-133.

Lawrence P and Weber L (2012) Midnight-2.00 a.m.: what goes on at the library? New Library World 113(11/12): 528-548.

Lippincott J (2010) Information commons: meeting millennials' needs. Journal of Library Administration 50(1): 27-37.

Ludwig L (2010) Health sciences libraries building survey, 1999-2009. Journal of the Medical Library Association 98(2): 105-134.

Martin LA (1976) User studies and library planning. Library Trends 24(3): 483-496.

May F and Swabey A (2015) Using and experiencing the academic library: a multi-site observational study of space and place. College \& Research Libraries. Epub ahead of print 10 November 2014. Manuscript number: cr114-683.

Montgomery SE (2014) Library space assessment: user learning behaviors in the library. The Journal of Academic Librarianship 40(1): 70-75.

Mozingo L (1989) Women and downtown open spaces. Places 6(1): 39-47.

Somerville MM and Brown-Sica M (2011) Library space planning: a participatory action research approach. The Electronic Library 29(5): 669-681.

Stewart C (2010) The Academic Library Building in the Digital Age: A Study of Construction, Planning, and Design of New Library Space. Chicago: Association of College and Research Libraries.

Vaska M, Chan R and Powelson S (2009) Results of a user survey to determine needs for a health sciences library renovation. New Review of Academic Librarianship 15(2): 219234. 
Wakaruk A (2009) Dissecting the disconnect: thinking about public space in academic libraries. College \& Research Libraries News 70(1): 16-19.

Waxman L, Clemons S, Banning J and McKelfresh D (2007) The library as place: providing students with opportunities for socialization, relaxation, and restoration. New Library World 108(9/10): 424-434.

Webb KM, Schaller MA and Hunley SA (2008) Measuring library space use and preferences: charting a path toward increased engagement. portal: Libraries and the Academy 8(4): 407-422.

Whitworth University Institutional Research (2015) Tenth day enrollment report spring 2015. Available at:

www.whitworth.edu/Administration/AcademicAffairs/InstitutionalResearch/FactBooks/ SpringFactbooks.html (accessed 5 March 2015).

Yoo-Lee EY, Lee TH, and Velez L (2013) Planning library spaces and services for millennials: an evidence-based approach. Library Management 34(6/7): 498-511. 